\title{
Total Extraperitoneal approach in large inguino-scrotal Hernias: an institutional approach
}

Tuhin Shah, Shah S, Joshi B R, Karkee R J, Gupta R K

Department of Surgery, B P. Koirala Institute of Health Sciences.

Correspondence: Tuhin Shah, Senior Resident

Department of Surgery, B P. Koirala Institute of Health Sciences

Email: dr.tuhinshah@gmail.com

\begin{abstract}
Introduction: Since 2 decades laparoscopichernia repair has gained key role in uncomplicated inguinal hernia surgery with advantages showed by several trials and guidelines. However, its role in complicated inguinal hernia such as incarcerated, obstructed and inguino-scrotal is debatable. Cases of large inguino-scrotal raises objection to laparoscopic procedure because of anticipated problems and complications in dissecting extended hernia sac even though posterior approach is advocated as repair of choice for complicated cases. Here, we reviewed our series of patients undergoing TEP in a limited time frame.
\end{abstract}

Method: Between March 2013 and June 2014, 50 consecutive patients underwent TEP repair for inguinoscrotal hernia. Patient demographics, hernia characteristics, operating time, surgical technique, conversion rate, intraoperative, postoperative complications and recurrence was recorded and analyzed using MS Excel.

Results: 50 patients were recorded, 26 had unilateral and 24 had bilateral hernias. Mean age was 52 (2272) years. The mean operation time was 70 (50-140) min. Bilateral repairs took $45 \%$ (18 min) longer than for unilateral repairs $(52+12 \mathrm{~min})$. Two (12.5\%) patientsrequired combined open surgery to transect the incarcerated omentum. There was no mortality. Morbidity was limited to asymptomatic seroma formation in 2 (12.5\%) patients; 1 patient of combined open-TEP approach had wound infection, both treated conservatively. Mean follow up was 6.3 months; we recorded 2 recurrences (12.5\%). The mean length of hospital stay was 1.8 days.

Conclusion: We conclude that TEP can be safely employed for complicated inguinal hernias repair. Surgical experience in mandatory with tailored technique to reduce morbidity and achieve good clinical outcome with acceptable recurrence rates.

Key words: Inguino-scrotal hernia; Total extraperitoneal hernia repair; Laparoscopy 